\title{
The Structure of Ionic Liquids at Electrified Interfaces - A Continuum Theory Based Approach
}

\author{
Max Schammer ${ }^{1,2^{*}}$, Birger Horstmann ${ }^{1,2,3}$, Arnulf Latz ${ }^{1,2,3}$ \\ ${ }^{1}$ Helmholtz Institute Ulm, Helmholtzstraße 11, 89081 Ulm, Germany \\ ${ }^{2}$ German Aerospace Center, Pfaffenwaldring 38, 70569 Stuttgart, Germany \\ ${ }^{3}$ University of Ulm, Albert-Einstein-Allee 47, $89081 \mathrm{Ulm}$, Germany
}

Ionic liquids (ILS) offer unique characteristics as battery electrolytes but are also highly interesting from a fundamentals point of view. Among these characteristics, the structure of the electrochemical double layer (EDL) is of significant importance.

Theoretical studies and simulations are efficient means for elucidating the conceptual mechanisms which govern the formation of the EDL-structures of ILs. Recently, we proposed a thermodynamic consistent transport theory for ILs and highly correlated electrolytes. ${ }^{[1]}$ This framework captures the dynamical description of IL-electrolytes by accounting for all relevant bulk-transport mechanisms. However, in order to describe highly charged regions in confined geometries, e.g. the EDL, we supplemented the continuum theory by non-local free energy contributions. These integral terms incorporate molecular interactions, which account for finite volume effects. In a joint experimental/theoretical publication, we validated this theoretical approach with AFM experiments. ${ }^{[2]}$ Thus, our approach offers a holistic framework for the description of IL-electrolytes in the bulk phase and near electrified interfaces, thus spanning a wide range of length-scales from cell-level (micrometers) to microscopic interactions (nanometers).

Here, we discuss the role of competing energy scales for the structure of the EDL. For this purpose, we supplement our numerical simulations by an analytic investigation of the integral formulation, using higher order gradients in the ion distribution. Thereby, we identify three energy scales related to short-ranged ion-correlations, accounting for excluded volume of hardcore ions, to the thermal energy, and to the electrostatic energy of Coulombic interactions. The relative magnitudes of these energy scales account for the resulting chargeprofile in the EDL. We predict the emergence of three screening-phases, consisting of a saturation-profile ("crowding"), followed by an exponentially decay towards the bulk, a profile of damped oscillations ("overscreening"), and a crystalline phase of undamped oscillations, which constitutes ion separation. Our analytic investigation gives precise predictions for the damping ratio and the frequency of the oscillations. We compare the analytic discussion with numerical simulations, and show that the analytic description is highly accurate.

In addition, we relate our holistic approach to alternative continuum models of the EDL for ILS (e.g., the BSK-formalism ${ }^{[3]}$ ). Furthermore, the integral formulation of our multiscale methodology relates directly to the methodology used by atomistic models like molecular dynamics simulations. 

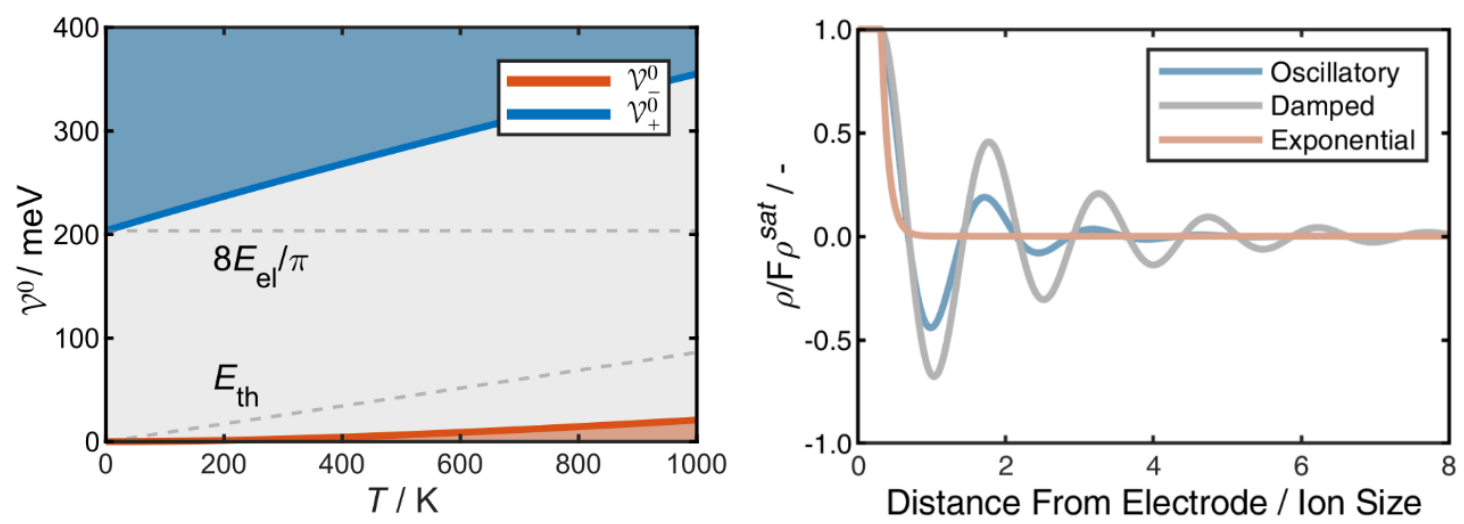

[1] Schammer, M., Horstmann, B., \& Latz, A. (2021). Journal of The Electrochemical Society, 168(2), 026511.

[2] Hoffmann, V., Pulletikurthi, G., Carstens, T., Lahiri, A., Borodin, A., Schammer, M., ... \& Endres, F. (2018). Physical Chemistry Chemical Physics, 20(7), 4760-4771.

[3] Bazant, M. Z., Storey, B. D., \& Kornyshev, A. A. (2011). Physical review letters, 106(4), 046102. 